\title{
HIGH RANK ELLIPTIC CURVES INDUCED BY RATIONAL DIOPHANTINE TRIPLES
}

\author{
Andrej Dujella and Juan Carlos Peral \\ University of Zagreb, Croatia, Universidad del País Vasco, Spain
}

Dedicated to the memory of our friend and coauthor Julián Aguirre

\begin{abstract}
A rational Diophantine triple is a set of three nonzero rational $a, b, c$ with the property that $a b+1, a c+1, b c+1$ are perfect squares. We say that the elliptic curve $y^{2}=(a x+1)(b x+1)(c x+1)$ is induced by the triple $\{a, b, c\}$. In this paper, we describe a new method for construction of elliptic curves over $\mathbb{Q}$ with reasonably high rank based on a parametrization of rational Diophantine triples. In particular, we construct an elliptic curve induced by a rational Diophantine triple with rank equal to 12 , and an infinite family of such curves with rank $\geq 7$, which are both the current records for that kind of curves.
\end{abstract}

\section{INTRODUCTION}

A set $\left\{a_{1}, a_{2}, \ldots, a_{m}\right\}$ of $m$ distinct nonzero rationals is called a rational Diophantine $m$-tuple if $a_{i} a_{j}+1$ is a perfect square for all $1 \leq i<j \leq$ $m$. The first rational Diophantine quadruple $\left\{\frac{1}{16}, \frac{33}{16}, \frac{17}{4}, \frac{105}{16}\right\}$ was found by Diophantus, while the first Diophantine quadruple in integers $\{1,3,8,120\}$ was found by Fermat. In 1969, Baker and Davenport ([2]) proved that Fermat's set cannot be extended to a Diophantine quintuple in integers. It was proved in [6] that there does not exist a Diophantine sextuple in integers and there are only finitely many Diophantine quintuples in integers. Recently, He, Togbé and Ziegler proved that there are no Diophantine quintuples in integers ([21]). Euler proved that there are infinitely many rational Diophantine quintuples. In particular, he extended Fermat's quadruple by the fifth positive rational number $\frac{777480}{8288641}$. In 2019, Stoll ([28]) proved that extension of Fermat's set

2020 Mathematics Subject Classification. 11G05, 11D09.

Key words and phrases. Elliptic curves, Diophantine triples, rank. 
to a rational quintuple with the same property is unique. The first example of a rational Diophantine sextuple, the set $\left\{\frac{11}{192}, \frac{35}{192}, \frac{155}{27}, \frac{512}{27}, \frac{1235}{48}, \frac{180873}{16}\right\}$, was found by Gibbs ([20]), while Dujella, Kazalicki, Mikić and Szikszai ([11]) recently proved that there are infinitely many rational Diophantine sextuples (see also $[10,12,13])$. For an overview of results on Diophantine $m$-tuples and its generalizations see [8].

Let $\{a, b, c\}$ be a rational Diophantine triple. Then there exist nonnegative rationals $r, s, t$ such that $a b+1=r^{2}, a c+1=s^{2}$ and $b c+1=t^{2}$. In order to extend the triple $\{a, b, c\}$ to a quadruple, we have to solve the system of equations

$$
a x+1=\square, \quad b x+1=\square, \quad c x+1=\square .
$$

We assign the following elliptic curve to the system (1.1):

$$
E: \quad y^{2}=(a x+1)(b x+1)(c x+1) .
$$

We say that the elliptic curve $E$ is induced by the rational Diophantine triple $\{a, b, c\}$.

Elliptic curves induced by rational Diophantine triples were used for the first time in the construction of elliptic curves with relatively large rank in [5] (let us mention that in [22] all $S$-integral points on some elliptic curves associated with the quintuple $\left\{\frac{1}{16}, \frac{33}{16}, \frac{105}{16}, 20,1140\right\}$ were computed, which was a motivation for considering connections between elliptic curves and Diophantine $m$-tuples). By using subtriples of certain rational Diophantine quintuples, elliptic curves with rank 7 over $\mathbb{Q}$ and rank 4 over $\mathbb{Q}(t)$ were constructed in [5]. That result was improved in [7] where several examples of curves with rank 9 were found by considering subtriples of the following generalization of Fermat's quadruple: $\left\{k-1, k+1,4 k, 16 k^{3}-4 k\right\}$. These results were further improved in our joint paper with Julián Aguirre ([1]), where we constructed an elliptic curve with rank 11 over $\mathbb{Q}$ (induced by the triple $\left.\left\{\frac{795025}{3128544},-\frac{22247424}{7791245}, \frac{24807390285149}{97501011189120}\right\}\right)$ and rank 5 over $\mathbb{Q}(t)$. The construction was based on subtriples of quadruples of the form $\left\{a, a(k+1)^{2}-2 k, a(2 k+\right.$ $\left.1)^{2}-8 k-4, a k^{2}-2 k-2\right\}$. We used similar method in [16] and constructed several new elliptic curves with rank 11 over $\mathbb{Q}$ and rank 6 over $\mathbb{Q}(t)$ (see also $[17])$.

Note that in all mentioned results the elliptic curves have torsion group $\mathbb{Z} / 2 \mathbb{Z} \times \mathbb{Z} / 2 \mathbb{Z}$. The application of elliptic curves induced by rational Diophantine triples in construction of high rank curves appears to be even more fruitful in the case of larger torsion groups. Such curves were used in $[15,17]$ for finding elliptic curves with the largest known rank over $\mathbb{Q}$ (rank 9; induced by the triples $\left\{-\frac{301273}{556614}, \frac{556614}{301273}, \frac{535707232}{290125899}\right\}$ and $\left.\left\{-\frac{181800}{127673}, \frac{127673}{181800}, \frac{996869751703}{2072406375000}\right\}\right)$ and $\mathbb{Q}(t)$ (rank 4 ) with torsion group $\mathbb{Z} / 2 \mathbb{Z} \times \mathbb{Z} / 4 \mathbb{Z}$. This construction uses triples of the form $\left\{a,-\frac{1}{a}, c\right\}$ which induce elliptic curves with points of order 4. It is shown in $[16]$ that the elliptic curve with largest known rank over $\mathbb{Q}$ 
(rank 6 ; originally found by Elkies in 2006) with torsion group $\mathbb{Z} / 2 \mathbb{Z} \times \mathbb{Z} / 6 \mathbb{Z}$ is induced by the triple $\left\{\frac{31269599}{31628160},-\frac{23721120}{31269599}, \frac{1461969791}{7144352640}\right\}$.

Furthermore, it was shown in [7] that every elliptic curve with torsion group $\mathbb{Z} / 2 \mathbb{Z} \times \mathbb{Z} / 8 \mathbb{Z}$ is induced by a Diophantine triple (see also $[3,14]$ ). In particular, the triple $\left\{\frac{408}{145},-\frac{145}{408},-\frac{145439}{59160}\right\}$ induces the curve with torsion group $\mathbb{Z} / 2 \mathbb{Z} \times \mathbb{Z} / 8 \mathbb{Z}$ and rank 3 over $\mathbb{Q}$, found by Connell and Dujella in 2000, what is the largest known rank for curves with that torsion group.

Although in the case of torsion group $\mathbb{Z} / 2 \mathbb{Z} \times \mathbb{Z} / 2 \mathbb{Z}$, the record ranks over $\mathbb{Q}($ rank 15$)$ and $\mathbb{Q}(t)$ (rank 7$)$ were discovered by Elkies $([18,19])$ with different methods, we believe that it is still interesting question to investigate how large can be the rank of elliptic curves induced by rational Diophantine triples. In this paper, we construct an elliptic curve induced by a rational Diophantine triples with rank equal to 12 , and an infinite family of such curves with rank $\geq 7$, which both improve previous results of the type.

\section{Construction of An Elliptic Curve With RANK 12}

By the coordinate transformation $x \mapsto \frac{x}{a b c}, y \mapsto \frac{y}{a b c}$, applied to the curve $E$, we obtain the equivalent curve

$$
E^{\prime}: \quad y^{2}=(x+a b)(x+a c)(x+b c) .
$$

The curve $E^{\prime}$ has three 2-rational points $A=[-b c, 0], B=[-a c, 0], C=$ $[-a b, 0]$, and other two rational points $P=[0, a b c]$ and $S=[1, r s t]$, where $a b+1=r^{2}, a c+1=s^{2}, b c+1=t^{2}$. We may expect that in general the points $P$ and $S$ will be independent points of infinite order, so that the rank of $E^{\prime}$ will be at least 2 .

To increase the rank, we will use the parametrization of rational Diophantine triples due to Lasić ([23]) (see also [13]):

$$
\begin{aligned}
a & =\frac{2 t_{1}\left(1+t_{1} t_{2}\left(1+t_{2} t_{3}\right)\right)}{\left(-1+t_{1} t_{2} t_{3}\right)\left(1+t_{1} t_{2} t_{3}\right)}, \\
b & =\frac{2 t_{2}\left(1+t_{2} t_{3}\left(1+t_{3} t_{1}\right)\right)}{\left(-1+t_{1} t_{2} t_{3}\right)\left(1+t_{1} t_{2} t_{3}\right)}, \\
c & =\frac{2 t_{3}\left(1+t_{3} t_{1}\left(1+t_{1} t_{2}\right)\right)}{\left(-1+t_{1} t_{2} t_{3}\right)\left(1+t_{1} t_{2} t_{3}\right)} .
\end{aligned}
$$

We have noted that the rank jumps if $t_{3}\left(t_{3}-t_{2}\right)$ is a perfect square (and, cyclicly, if $t_{1}\left(t_{1}-t_{3}\right)$ is a perfect square or if $t_{2}\left(t_{2}-t_{1}\right)$ is a perfect square). Indeed, if we insert

$$
x=-\frac{4\left(t_{2}^{2} t_{3}-t_{3}+t_{2}\right)\left(t_{3} t_{1}^{2} t_{2}+1+t_{3} t_{1}\right)\left(t_{2} t_{3}+t_{2} t_{3}^{2} t_{1}+1\right)}{t_{3}\left(-1+t_{1} t_{2} t_{3}\right)^{2}\left(1+t_{1} t_{2} t_{3}\right)^{2}}
$$


(note that $x+a b=\frac{b(c-b)}{t_{2} t_{3}}$ ) into the equation (2.1), we obtain

$$
\begin{aligned}
y^{2}= & 64\left(1+t_{3} t_{1}\right)^{2}\left(t_{1} t_{2} t_{3}-t_{2}-t_{2}^{2} t_{3}+t_{3}\right)^{2}\left(t_{2} t_{3}+t_{2} t_{3}^{2} t_{1}+1\right)^{2}\left(1+t_{2} t_{3}\right)^{2} \\
& \times\left(t_{3} t_{1}^{2} t_{2}+1+t_{3} t_{1}\right)^{2}\left(t_{3}-t_{2}\right) t_{3}^{-3}\left(-1+t_{1} t_{2} t_{3}\right)^{-6}\left(1+t_{1} t_{2} t_{3}\right)^{-6},
\end{aligned}
$$

which leads to the condition that $t_{3}\left(t_{3}-t_{2}\right)$ is a perfect square.

Thus, if we find a triple $\left(t_{1}, t_{2}, t_{3}\right)$ of rationals such that

$$
t_{3}\left(t_{3}-t_{2}\right), \quad t_{1}\left(t_{1}-t_{3}\right), \quad t_{2}\left(t_{2}-t_{1}\right)
$$

are all perfect squares, we may expect that our curve will have rank $\geq 5$ (since we started with rank $\geq 2$ ).

One way to satisfy conditions (2.2) is through so called almost perfect cuboids. Indeed, if we put

$$
t_{3}=s_{3}^{2}, t_{1}=-s_{1}^{2}, t_{2}=s_{2}^{2}, s_{3}^{2}-s_{2}^{2}=s_{4}^{2},
$$

then we have

$$
s_{1}^{2}+s_{2}^{2}=\mathrm{\square}, s_{2}^{2}+s_{4}^{2}=\mathrm{\square}, s_{1}^{2}+s_{2}^{2}+s_{4}^{2}=\mathbf{\square} .
$$

Thus we get an almost perfect cuboid (only one diagonal is not an integer). In [29], one can find a parametric solution of (2.3):

$$
\begin{aligned}
& s_{1}=2\left(m^{2}+m+1\right)\left(m^{2}-1\right)^{2}\left(m^{2}+1+4 m\right), \\
& s_{2}=4\left(m^{2}+m+1\right)(2 m+1)\left(m^{2}-1\right)\left(2 m+m^{2}\right), \\
& s_{4}=(2 m+1)\left(2 m+m^{2}\right)\left(3 m^{2}+2 m+1\right)\left(m^{2}+2 m+3\right),
\end{aligned}
$$

which gives

$$
\begin{aligned}
& t_{1}=-4\left(m^{2}+m+1\right)^{2}\left(m^{2}-1\right)^{4}\left(m^{2}+1+4 m\right)^{2}, \\
& t_{2}=16\left(m^{2}+m+1\right)^{2}(2 m+1)^{2}\left(m^{2}-1\right)^{2}\left(2 m+m^{2}\right)^{2}, \\
& t_{3}=m^{2}(2 m+1)^{2}(m+2)^{2}\left(5 m^{2}+8 m+5\right)^{2}\left(m^{2}+1\right)^{2} .
\end{aligned}
$$

We now present another approach which yields a two-parametric solution, more appropriate for numerical experiments for finding specializations with higher rank. We satisfy the first two conditions by putting

$$
t_{3}\left(t_{3}-t_{2}\right)=\left(t_{3}+u\right)^{2}, \quad t_{1}\left(t_{1}-t_{3}\right)=\left(t_{1}+v\right)^{2}
$$

and we get

$$
t_{2}=-\frac{u\left(2 t_{3}+u\right)}{t_{3}}, \quad t_{3}=-\frac{v\left(2 t_{1}+v\right)}{t_{1}} .
$$

By inserting this into the third condition $t_{2}\left(t_{2}-t_{1}\right)=\mathrm{Q}$, we get

$$
\begin{aligned}
& \left(8 u v^{2}-2 u^{2} v\right) t_{1}^{3}+\left(-8 u^{3} v+15 u^{2} v^{2}+u^{4}+8 u v^{3}\right) t_{1}^{2} \\
& +\left(-4 u^{3} v^{2}+2 v^{4} u+16 u^{2} v^{3}\right) t_{1}+4 v^{4} u^{2}=\mathrm{\square} .
\end{aligned}
$$


The equation $(2.4)$ can be viewed as an elliptic curve over $\mathbb{Q}(u, v)$, with an obvious point $P=\left[0,2 u^{2} v^{2}\right]$. By taking the point $2 P$, we obtain

$$
t_{1}=\frac{v^{2}(-v+16 u)}{8 u(-4 v+u)}
$$

which gives

$$
\begin{aligned}
a & =-\frac{v^{2}(-v+16 u)\left(16 v^{2}-64 u^{2}-v^{4}+16 u v^{3}-4 v^{5} u+v^{4} u^{2}\right)}{u(2+v)\left(4-2 v+v^{2}\right)(v-2)\left(v^{2}+2 v+4\right)(2 u-v)(2 u+v)(-4 v+u)}, \\
b & =\frac{16 u(-4 v+u) v\left(4 v-64 u+16 u v^{2}-4 u^{2} v-v^{5}+4 u^{2} v^{3}\right)}{(2+v)\left(4-2 v+v^{2}\right)(v-2)\left(v^{2}+2 v+4\right)(2 u-v)(2 u+v)(-v+16 u)}, \\
c & =\frac{4\left(256 u v-64 u^{2}-16 v^{4}+64 u^{2} v^{2}+v^{6}-16 v^{5} u\right)(2 u-v)(2 u+v)}{u(2+v)\left(4-2 v+v^{2}\right)(v-2)\left(v^{2}+2 v+4\right)(-v+16 u)(-4 v+u)} .
\end{aligned}
$$

This gives the elliptic curve with rank $\geq 5$ over $\mathbb{Q}(u, v)$. Indeed, if we write the curve in the form $y^{2}=x^{3}+A x^{2}+\bar{B} x$, where

$$
\begin{aligned}
A= & v\left(256 v^{13}-32 v^{15}+v^{17}+140288 v^{9} u^{2}+741888 v^{7} u^{4}-4096 v^{10} u-1167360 v^{8} u^{3}\right. \\
& -21258240 v^{6} u^{5}-7936 v^{12} u+664832 v^{10} u^{3}+11440128 v^{8} u^{5}+32192 v^{11} u^{2} \\
& -2785824 v^{9} u^{4}-32380416 v^{7} u^{6}+28747776 v^{5} u^{6}+6463488 v^{6} u^{7}+71860224 u^{7} v^{4} \\
& -2205696 u^{8} v^{5}+1536 v^{14} u-24192 v^{13} u^{2}-22528 v^{12} u^{3}+591360 v^{11} u^{4} \\
& -3244800 u^{5} v^{10}-128483328 v^{3} u^{8}-12979200 v^{8} u^{7}+7816 v^{15} u^{2}-36160 v^{14} u^{3} \\
& -8616 v^{13} u^{4}+100992 v^{12} u^{5}-128 v^{16} u-2023776 v^{11} u^{6}+4 v^{18} u-449 v^{17} u^{2} \\
& +7824 v^{16} u^{3}-31368 v^{15} u^{4}+2860032 v^{10} u^{7}+70176 v^{14} u^{5}+112296 v^{1} 3 u^{6} \\
& +9461760 v^{7} u^{8}-2785824 v^{9} u^{8}-332160 v^{12} u^{7}+128188416 v^{2} u^{9}-37027840 v^{4} u^{9} \\
& -1441792 v^{6} u^{9}+2659328 v^{8} u^{9}+46368 v^{11} u^{8}-6193152 u^{10} v^{5}+515072 u^{10} v^{7} \\
& -291840 u^{9} v^{10}+16818240 v^{9} u^{6}-29425664 v u^{10}+32014336 u^{10} v^{3}+140288 v^{9} u^{10} \\
& -2097152 u^{11} v^{2}+1572864 u^{11} v^{4}-507904 u^{11} v^{6}-16384 u^{11} v^{8}+65536 u^{12} v^{5} \\
& \left.+65536 u^{12} v-131072 u^{12} v^{3}+1048576 u^{11}\right), \\
B= & 4\left(8 v u^{2}-8 u^{2}+16 v u-v^{2} u+v^{2}+2 v^{3}\right)\left(8 v u^{2}+8 u^{2}-16 v u-v^{2} u-v^{2}+2 v^{3}\right) \\
& \times\left(-16 v^{2}+64 u^{2}+v^{4}-16 v^{3} u\right)\left(4 v-64 u+16 v^{2} u-4 v u^{2}-v^{5}+4 v^{3} u^{2}\right) \\
& \times\left(2 v u^{2}-16 u^{2}+2 v u+8 v^{2} u-4 v^{2}-v^{3}\right)\left(16 v u-4 u^{2}-v^{4}+4 v^{2} u^{2}\right) \\
& \times\left(16 v^{2}-64 u^{2}-v^{4}+16 v^{3} u-4 v^{5} u+v^{4} u^{2}\right) \\
& \times\left(2 v u^{2}+16 u^{2}-2 v u+8 v^{2} u+4 v^{2}-v^{3}\right)(-v+16 u)^{2}(-4 v+u)^{2} u^{2} v^{3},
\end{aligned}
$$


then five independent points of infinite order are

$$
\begin{aligned}
& P=\left[-4\left(4 v-64 u+16 v^{2} u-4 v u^{2}-v^{5}+4 v^{3} u^{2}\right)(-4 v+u)^{2}(-v+16 u)^{2}\right. \\
& \times\left(16 v^{2}-64 u^{2}-v^{4}+16 v^{3} u-4 v^{5} u+v^{4} u^{2}\right) u^{2} v^{3}, \\
& 8\left(64 v^{2} u^{2}-64 u^{2}-16 v^{5} u+256 v u+v^{6}-16 v^{4}\right)\left(4 v-64 u+16 v^{2} u-4 v u^{2}-v^{5}+4 v^{3} u^{2}\right) \\
& \times\left(16 v^{2}-64 u^{2}-v^{4}+16 v^{3} u-4 v^{5} u+v^{4} u^{2}\right)(2 u-v)^{2}(2 u+v)^{2}(-4 v+u)^{2} \\
& \left.\times(-v+16 u)^{2} u^{2} v^{3}\right], \\
& R=\left[4\left(16 v u-4 u^{2}-v^{4}+4 v^{2} u^{2}\right)\left(16 v^{2}-64 u^{2}-v^{4}+16 v^{3} u-4 v^{5} u+v^{4} u^{2}\right)\right. \\
& \times\left(8 v u^{2}-8 u^{2}+16 v u-v^{2} u+v^{2}+2 v^{3}\right)\left(8 v u^{2}+8 u^{2}-16 v u-v^{2} u-v^{2}+2 v^{3}\right) \\
& \times(-v+16 u)(-4 v+u) v u, \\
& 4\left(8 v u^{2}-8 u^{2}+16 v u-v^{2} u+v^{2}+2 v^{3}\right)\left(16 v^{2}-64 u^{2}-v^{4}+16 v^{3} u-4 v^{5} u+v^{4} u^{2}\right) \\
& \times\left(8 v u^{2}+8 u^{2}+32 v u-16 v^{2} u-4 v^{2}-v^{3}\right)\left(16 v u-4 u^{2}-v^{4}+4 v^{2} u^{2}\right) \\
& \times\left(8 v u^{2}+8 u^{2}-16 v u-v^{2} u-v^{2}+2 v^{3}\right)\left(8 v u^{2}-8 u^{2}-32 v u-16 v^{2} u+4 v^{2}-v^{3}\right) \\
& \left.\times(2 u+v)(2 u-v) v^{2}(-v+16 u)(-4 v+u) u\right], \\
& T_{1}=\left[16\left(16 v u-4 u^{2}-v^{4}+4 v^{2} u^{2}\right)\left(2 v u^{2}-16 u^{2}+2 v u+8 v^{2} u-4 v^{2}-v^{3}\right)\right. \\
& \times\left(2 v u^{2}+16 u^{2}-2 v u+8 v^{2} u+4 v^{2}-v^{3}\right)\left(4 v-64 u+16 v^{2} u-4 v u^{2}-v^{5}+4 v^{3} u^{2}\right) \\
& \times(-v+16 u)(-4 v+u) u, \\
& 8\left(16 v u-4 u^{2}-v^{4}+4 v^{2} u^{2}\right)\left(2 v u^{2}-16 u^{2}+2 v u+8 v^{2} u-4 v^{2}-v^{3}\right) \\
& \times\left(2 v u^{2}+16 u^{2}-2 v u+8 v^{2} u+4 v^{2}-v^{3}\right)\left(-v+16 u-4 v^{2} u+v u^{2}\right) \\
& \times\left(v^{6}-16 v^{5} u+256 v u-64 u^{2}-16 v^{4}+64 v^{2} u^{2}\right)\left(8 u^{2}-v u+2 v^{2}\right) \\
& \left.\times\left(4 v-64 u+16 v^{2} u-4 v u^{2}-v^{5}+4 v^{3} u^{2}\right)(-v+16 u)(-4 v+u) u\right], \\
& T_{2}=\left[-4\left(8 v u^{2}-8 u^{2}+16 v u-v^{2} u+v^{2}+2 v^{3}\right)\left(-16 v^{2}+64 u^{2}+v^{4}-16 v^{3} u\right)\right. \\
& \times\left(16 v u-4 u^{2}-v^{4}+4 v^{2} u^{2}\right)\left(8 v u^{2}+8 u^{2}-16 v u-v^{2} u-v^{2}+2 v^{3}\right) \\
& \times\left(16 v^{2}-64 u^{2}-v^{4}+16 v^{3} u-4 v^{5} u+v^{4} u^{2}\right)(-4 v+u) u / v^{2}, \\
& 4\left(8 v u^{2}-8 u^{2}+16 v u-v^{2} u+v^{2}+2 v^{3}\right)\left(8 v u^{2}+8 u^{2}-16 v u-v^{2} u-v^{2}+2 v^{3}\right) \\
& \times\left(16 v u-4 u^{2}-v^{4}+4 v^{2} u^{2}\right)\left(-16 v^{2}+64 u^{2}+v^{4}-16 v^{3} u\right)(2 u+v)(2 u-v) \\
& \times\left(8 u^{2}-16 v u-v^{2}\right)\left(-16 v^{4}+64 v^{2} u^{2}+v^{6}-16 v^{5} u+256 v u-64 u^{2}\right) \\
& \left.\times\left(16 v^{2}-64 u^{2}-v^{4}+16 v^{3} u-4 v^{5} u+v^{4} u^{2}\right)(-4 v+u) u / v^{3}\right], \\
& T_{3}=\left[\left(4 v-64 u+16 v^{2} u-4 v u^{2}-v^{5}+4 v^{3} u^{2}\right)\left(-16 v^{2}+64 u^{2}+v^{4}-16 v^{3} u\right)\right. \\
& \times\left(16 v^{2}-64 u^{2}-v^{4}+16 v^{3} u-4 v^{5} u+v^{4} u^{2}\right)\left(2 u^{2}+8 v u-v^{2}\right)^{2}(-v+16 u), \\
& 2\left(-16 v^{2}+64 u^{2}+v^{4}-16 v^{3} u\right)\left(8 v u^{2}-8 u^{2}-32 v u-16 v^{2} u+4 v^{2}-v^{3}\right) \\
& \times\left(8 v u^{2}+8 u^{2}+32 v u-16 v^{2} u-4 v^{2}-v^{3}\right)\left(-v+16 u-4 v^{2} u+v u^{2}\right) \\
& \times\left(16 v^{2}-64 u^{2}-v^{4}+16 v^{3} u-4 v^{5} u+v^{4} u^{2}\right)\left(2 u^{2}+8 v u-v^{2}\right) \\
& \left.\times\left(4 v-64 u+16 v^{2} u-4 v u^{2}-v^{5}+4 v^{3} u^{2}\right)(2 u-v)(2 u+v)(-v+16 u)\right] .
\end{aligned}
$$


Here the point $P$ corresponds to $[0, a b c]$ on $y^{2}=(x+a b)(x+a c)(x+b c)$, the point $R$ satisfies $2 R=S$, where $S$ corresponds to $[1, r s t]$ on $y^{2}=(x+$ $a b)(x+a c)(x+b c)$, the point $T_{1}$ corresponds to the condition $t_{3}\left(t_{3}-t_{2}\right)=\mathrm{\square}$, the point $T_{2}$ corresponds to the condition $t_{1}\left(t_{1}-t_{3}\right)=\mathrm{D}$, while the point $T_{3}$ corresponds to the condition $t_{2}\left(t_{2}-t_{1}\right)=\square$. Since the specialization map in a homomorphism, it suffices to find a specialization $\left(u_{0}, v_{0}\right)$ for which the points $P, R, T_{1}, T_{2}$ and $T_{3}$ are independent points of infinite order on $y^{2}=x^{3}+A x^{2}+B x$. We checked that this is the case for $\left(u_{0}, v_{0}\right)=(2,1)$, since the points [170605, 39532697], [302665, -66247363], [795565,-637321303], [-447095,-24260803], [8673115/4,-25165674989/8] are independent on $y^{2}=x^{3}+21361758597 x^{2}-28803989016278714304 x$.

Now we search for specializations $(u, v)$ with higher rank, in particular with rank 11 and 12 . We use a sieving methods similar to those used, e.g., in $[1,16]$. We searched for curves with relatively large Mestre-Nagao sum

$$
S(N, E)=\sum_{p=2}^{N} \frac{-a_{p}+2}{p+1-a_{p}} \log p,
$$

where $a_{p}=a_{p}(E)=p+1-\# E\left(\mathbb{F}_{p}\right)$, since it is experimentally known $([24,25])$ that we may expect that high rank curves have large $S(N, E)$, and large Selmer rank (as implemented in mwrank with option -s). In search for rank 12 curves we also use the condition that the root-number is equal to 1 (conjecturally this implies that rank is even). We searched also in some restricted subfamilies, including e.g. $u=v$. We implemented the sieving algorithm in Pari ([26]). For the curves which pass our searching conditions, we calculate the rank by Cremona's program mwrank ([4]).

We find curves with rank 11 for the following parameters:

$$
\begin{aligned}
& (u, v)= \\
& \left(\frac{11}{24}, \frac{5}{9}\right),\left(-\frac{145}{6}, \frac{29}{12}\right),\left(\frac{136}{19}, \frac{68}{5}\right),\left(-\frac{16}{77}, \frac{4}{21}\right),\left(\frac{473}{705}, \frac{43}{47}\right),\left(-\frac{89}{135}, \frac{89}{45}\right), \\
& \left(-\frac{62}{43}, \frac{93}{43}\right),\left(\frac{71}{273}, \frac{142}{91}\right),\left(\frac{224}{67}, \frac{7}{2}\right),\left(-\frac{1032}{923}, \frac{172}{71}\right),\left(-\frac{1501}{87}, \frac{158}{87}\right), \\
& \left(\frac{1358}{1007}, \frac{194}{53}\right),\left(-\frac{2072}{1819}, \frac{148}{107}\right),\left(\frac{454}{481}, \frac{227}{37}\right),\left(\frac{77}{173}, \frac{77}{173}\right),\left(\frac{163}{137}, \frac{163}{137}\right) .
\end{aligned}
$$

The details (minimal Weierstrass equation, torsion points and independent points of infinite order) are given in [9]. Let us mention that the curve corresponding to $(u, v)=\left(-\frac{62}{43}, \frac{93}{43}\right)$, i.e.

$$
\{a, b, c\}=\left\{\frac{21409906185}{74591676404},-\frac{31580198976}{18647919101},-\frac{10309975195}{18647919101}\right\},
$$


with the minimal Weierstrass equation

$$
\begin{aligned}
y^{2}+x y= & x^{3}-x^{2}-21252276640652798739707819217 x \\
& +938627524108684110053910801619511357084941,
\end{aligned}
$$

has the minimal discriminant among all known curves with rank 11 and torsion group $\mathbb{Z} / 2 \mathbb{Z} \times \mathbb{Z} / 2 \mathbb{Z}$.

Finally, we found a curve with rank 12 for $(u, v)=\left(-\frac{95}{33}, \frac{50}{57}\right)$, i.e.

$$
\{a, b, c\}=\left\{\frac{6125241375}{11907531272}, \frac{5535371271425}{14277129995128},-\frac{273138178560}{153430695649}\right\},
$$

with the minimal Weierstrass equation

$$
\begin{aligned}
& y^{2}+x y+y=x^{3}-x^{2}-1444491707528591356856089186460491195711268950880 x \\
& +559921583779625421248683584939561762456224290170437461555851482041439747,
\end{aligned}
$$

the torsion points

$$
\begin{aligned}
& \mathcal{O},[910954389920845836020349,-455477194960422918010175], \\
& {[-5448727291190824028230629 / 4,5448727291190824028230625 / 8],} \\
& {[451227432876860171037309,-225613716438430085518655],}
\end{aligned}
$$

and 12 independent points of infinite order

$$
\begin{aligned}
P_{1}= & {[158850932500649609134809,578334775816714524616276221704042845], } \\
P_{2}= & {[351104017200784386392209,309897966944945116194624198332593845], } \\
P_{3}= & {[-427722660290928813983135,-1048576645526111528109185629948786727], } \\
P_{4}= & {[954500781939375762742909,225326008863345220543071618783370945], } \\
P_{5}= & {[423679598259676591990909,154829810959547852593332987635966145], } \\
P_{6}= & {[1535808449095818094207905,1401421444080498380369785533616999513], } \\
P_{7}= & {[444801887422056021535383,73569216148613399817347986859758945], } \\
P_{8}= & {[-1206006015871044278678751,-740210245609217615143269452335454375], } \\
P_{9}= & {[-192562292438693523617091,-911556889640548767064630159456313855], } \\
P_{10}= & {[10508879668527356682921249,33851800053181168926568362825476385625], } \\
P_{11}= & {[951514410733369555670349,216676520921276805299703311439049825], } \\
P_{12}= & {[-7355680099955426717481581 / 81,} \\
& -605705671933225602690651446390633849125 / 729] .
\end{aligned}
$$

Let us also mention a minor, somewhat related result: for $t_{1}=\frac{44}{29}, t_{2}=$ $\frac{17}{42}, t_{3}=\frac{3}{44}$, i.e. $a=\frac{815848}{164547}, b=\frac{1512524}{1810017}, c=\frac{32060}{201113}$, we get the elliptic curve

$$
\begin{aligned}
y^{2}= & x^{3}+x^{2}-193936360896469946772176 x \\
& +29453641253718130506136229522416740
\end{aligned}
$$

with rank 10, which is the curve with smallest known conductor among curves with rank 10 and torsion group $\mathbb{Z} / 2 \mathbb{Z} \times \mathbb{Z} / 2 \mathbb{Z}$. It is obtained by brute-force 
search (not in parametric families) within triples $t_{1}, t_{2}, t_{3}$ with small numerators and denominators.

\section{INFINITE FAMILIES OF ELLIPTIC CURVES WITH RANK $\geq 7$}

The construction of the two-parametric family of curves with rank $\geq 5$ from the previous section is related with the construction from our joint paper with Julián Aguirre ([1]). In [1], we constructed a two-parametric family of curves with rank $\geq 4$ over $\mathbb{Q}(m, n)$, and by choosing $n=7 / 3$ we obtained a family with rank $\geq 5$ over $\mathbb{Q}(m)$. It can be checked that by taking $m=$ $-\frac{20\left(4 u^{2}-1\right)}{9 u(u+4)}$ we obtain the same family as the family obtained from our new two-parametric family by specializing $v=-1$.

It is shown in [16] that inserting $n=7 / 3$ already in the family from [1] with rank $\geq 3$ over $\mathbb{Q}(a, n)$, gives a simple family with rank $\geq 4$ over $\mathbb{Q}(a)$, which is very suitable for constructing subfamilies with higher rank. That family is

$$
y^{2}=x^{3}+A(a) x^{2}+B(a) x,
$$

where

$$
\begin{aligned}
& A(a)=-2\left(-51200+109440 a+38880 a^{2}+55404 a^{3}+6561 a^{4}\right), \\
& B(a)=243 a^{2}(20+3 a)(-4+9 a)(16+9 a)(80+9 a)\left(320+81 a^{2}\right),
\end{aligned}
$$

and the $x$-coordinates of four independent points of infinite order are

$$
\begin{aligned}
& x_{1}=81 a^{2}(-4+9 a)(80+9 a), \\
& x_{2}=27 a(20+3 a)(-4+9 a)(80+9 a), \\
& x_{3}=\frac{1}{441}(-4+9 a)(80+9 a)(160+171 a)^{2}, \\
& x_{4}=3(20+3 a)(-4+9 a)\left(320+81 a^{2}\right) .
\end{aligned}
$$

There are several substitutions which give subfamilies with rank $\geq 6$ :

$$
\begin{aligned}
& a=-\frac{2\left(-27+13 w_{1}^{2}\right)\left(-13+27 w_{1}^{2}\right)}{9\left(9+178 w_{1}^{2}+9 w_{1}^{4}\right)}, \\
& a=-\frac{64\left(831744-40128 w_{2}+4288 w_{2}^{2}-44 w_{2}^{3}+w_{2}^{4}\right)}{9\left(-1520+88 w_{2}+w_{2}^{2}\right)\left(-2736-264 w_{2}+5 w_{2}^{2}\right)}, \\
& a=\frac{10732176+628992 w_{3}+19192 w_{3}^{2}+576 w_{3}^{3}+9 w_{3}^{4}}{36 w_{3}\left(27+w_{3}\right)\left(364+9 w_{3}\right)}, \\
& a=\frac{5\left(-10+6 w_{4}+w_{4}^{2}\right)\left(-18-18 w_{4}+5 w_{4}^{2}\right)}{9\left(12-2 w_{4}+w_{4}^{2}\right)\left(3-w_{4}+w_{4}^{2}\right)}, \\
& a=\frac{5\left(584820+135432 w_{5}-18288 w_{5}^{2}+396 w_{5}^{3}+5 w_{5}^{4}\right)}{9\left(684-66 w_{5}+w_{5}^{2}\right)\left(171-33 w_{5}+w_{5}^{2}\right)} .
\end{aligned}
$$


The first four substitutions were already given in [16], while the fifth substitution is new.

In order to find infinite families with rank $\geq 7$, we try to find intersections of these five families with rank $\geq 6$. We compare their $j$-invariants by factorizing their difference and seeking for the factors which correspond to curves with genus 1 .

If we compare the second and third substitution, we find two suitable factors, which give the following conditions:

$$
\begin{aligned}
& w_{2}^{2} w_{3}^{2}+72 w_{2}^{2} w_{3}+88 w_{2} w_{3}^{2}+1820 w_{2}^{2}-1520 w_{3}^{2} \\
& \quad-96096 w_{2}-65664 w_{3}-995904=0, \\
& \quad 5 w_{2}^{2} w_{3}^{2}+216 w_{2}^{2} w_{3}-264 w_{2} w_{3}^{2}+3276 w_{2}^{2}-2736 w_{3}^{2} \\
& \quad+288288 w_{2}-196992 w_{3}-4979520=0 .
\end{aligned}
$$

Both conditions lead to

$$
54 w_{3}^{4}+2736 w_{3}^{3}+66592 w_{3}^{2}+2987712 w_{3}+64393056=\square .
$$

This quartic is birationally equivalent to the elliptic curve

$$
y^{2}=x^{3}+x^{2}-28174550 x+45644288448
$$

with rank equal to 3 , hence the elliptic curve, and also the quartic, have infinitely many rational solutions. Many of them produce curves with rank $=7$, e.g. $w_{3}=-234,-30,-18,26,42,94,-\frac{202}{3},-\frac{182}{3},-\frac{14}{3}$.

Consider the four points given by (3.1) and additional two points corresponding to the second and third substitutions. The second substitution gives the curve

$$
y^{2}=x^{3}+a_{62} x^{2}+b_{62} x
$$

where

$$
\begin{aligned}
a_{62}= & 79573 w_{2}^{16}+2281840 w_{2}^{15}-791687936 w_{2}^{14}-34844285696 w_{2}^{13} \\
& +3065917324288 w_{2}^{12}+556971294060544 w_{2}^{11}-64165839736733696 w_{2}^{10} \\
& +3360211454234263552 w_{2}^{9}-130403990149389221888 w_{2}^{8} \\
& +3064512846261648359424 w_{2}^{7}-53369552205989831245824 w_{2}^{6} \\
& +422490869190468915167232 w_{2}^{5}+2120995723090424777146368 w_{2}^{4} \\
& -21983951517250398896259072 w_{2}^{3}-455536370311599498486349824 w_{2}^{2} \\
& +1197427029434259336824094720 w_{2}+38082411231292796255084740608
\end{aligned}
$$




$$
\begin{aligned}
b_{62}= & -5184\left(w_{2}^{4}-44 w_{2}^{3}+4288 w_{2}^{2}-40128 w_{2}+831744\right)^{2} \\
& \times\left(w_{2}^{4}+352 w_{2}^{3}-50720 w_{2}^{2}+321024 w_{2}+831744\right) \\
& \times\left(3 w_{2}^{4}+352 w_{2}^{3}+15328 w_{2}^{2}-642048 w_{2}+5822208\right) \\
& \times\left(7 w_{2}^{4}-704 w_{2}^{3}+15328 w_{2}^{2}+321024 w_{2}+2495232\right) \\
& \times\left(7 w_{2}^{4}-176 w_{2}^{3}+11680 w_{2}^{2}-160512 w_{2}+5822208\right) \\
& \times\left(7 w_{2}^{4}+352 w_{2}^{3}-61664 w_{2}^{2}+321024 w_{2}+5822208\right) \\
& \times\left(59 w_{2}^{4}+3344 w_{2}^{3}-572128 w_{2}^{2}+3049728 w_{2}+49072896\right),
\end{aligned}
$$

and six independent points of infinite order with $x$-coordinates:

$$
\begin{aligned}
x_{21}= & -576\left(w_{2}^{4}-44 w_{2}^{3}+4288 w_{2}^{2}-40128 w_{2}+831744\right)^{2} \\
& \times\left(7 w_{2}^{4}-176 w_{2}^{3}+11680 w_{2}^{2}-160512 w_{2}+5822208\right) \\
& \times\left(7 w_{2}^{4}+352 w_{2}^{3}-61664 w_{2}^{2}+321024 w_{2}+5822208\right), \\
x_{22}= & 36\left(w_{2}^{4}-44 w_{2}^{3}+4288 w_{2}^{2}-40128 w_{2}+831744\right) \\
& \times\left(7 w_{2}^{4}-176 w_{2}^{3}+11680 w_{2}^{2}-160512 w_{2}+5822208\right) \\
& \times\left(7 w_{2}^{4}+352 w_{2}^{3}-61664 w_{2}^{2}+321024 w_{2}+5822208\right) \\
& \times\left(59 w_{2}^{4}+3344 w_{2}^{3}-572128 w_{2}^{2}+3049728 w_{2}+49072896\right), \\
x_{23}= & -16 / 49\left(7 w_{2}^{4}-176 w_{2}^{3}+11680 w_{2}^{2}-160512 w_{2}+5822208\right) \\
& \times\left(7 w_{2}^{4}+352 w_{2}^{3}-61664 w_{2}^{2}+321024 w_{2}+5822208\right) \\
& \times\left(13 w_{2}^{4}-2552 w_{2}^{3}+330784 w_{2}^{2}-2327424 w_{2}+10812672\right)^{2}, \\
x_{24}= & -27 / 4\left(3 w_{2}^{4}+352 w_{2}^{3}+15328 w_{2}^{2}-642048 w_{2}+5822208\right) \\
& \times\left(7 w_{2}^{4}-704 w_{2}^{3}+15328 w_{2}^{2}+321024 w_{2}+2495232\right) \\
& \times\left(7 w_{2}^{4}-176 w_{2}^{3}+11680 w_{2}^{2}-160512 w_{2}+5822208\right) \\
& \times\left(59 w_{2}^{4}+3344 w_{2}^{3}-572128 w_{2}^{2}+3049728 w_{2}+49072896\right), \\
x_{25}= & -108\left(w_{2}^{2}-912\right)^{2}\left(w_{2}^{4}+352 w_{2}^{3}-50720 w_{2}^{2}+321024 w_{2}+831744\right) \\
& \times\left(7 w_{2}^{4}+352 w_{2}^{3}-61664 w_{2}^{2}+321024 w_{2}+5822208\right) \\
& \times\left(59 w_{2}^{4}+3344 w_{2}^{3}-572128 w_{2}^{2}+3049728 w_{2}+49072896\right), \\
x_{26}= & 324\left(w_{2}^{2}-912\right)^{2}\left(w_{2}^{4}+352 w_{2}^{3}-50720 w_{2}^{2}+321024 w_{2}+831744\right) \\
& \times\left(7 w_{2}^{4}-176 w_{2}^{3}+11680 w_{2}^{2}-160512 w_{2}+5822208\right) \\
& \times\left(7 w_{2}^{4}+352 w_{2}^{3}-61664 w_{2}^{2}+321024 w_{2}+5822208\right) . \\
&
\end{aligned}
$$

The third condition gives the curve

$$
y^{2}=x^{3}+a_{63} x^{2}+b_{63} x,
$$


where

$$
\begin{aligned}
a_{63}= & -13122 w_{3}^{16}-7348320 w_{3}^{15}-1570137696 w_{3}^{14}-206172584064 w_{3}^{13} \\
& -19541430237312 w_{3}^{12}-1402008391816704 w_{3}^{11}-77606011598363136 w_{3}^{10} \\
& -3410103604914358272 w_{3}^{9}-123219415654113963008 w_{3}^{8} \\
& -3723833136566479233024 w_{3}^{7}-92542375014630498607104 w_{3}^{6} \\
& -1825654232153731017572352 w_{3}^{5}-27787335201034030779236352 w_{3}^{4} \\
& -320143070559304939026382848 w_{3}^{3}-2662401630093588063697895424 w_{3}^{2} \\
& -13606503227295711027839631360 w_{3}-26532681293226636504287281152, \\
b_{63}= & 81\left(w_{3}^{4}+72 w_{3}^{3}+8504 w_{3}^{2}+550368 w_{3}+10732176\right) \\
& \times\left(3 w_{3}^{4}+144 w_{3}^{3}+3160 w_{3}^{2}+157248 w_{3}+3577392\right) \\
& \times\left(3 w_{3}^{4}+1152 w_{3}^{3}+71144 w_{3}^{2}+1257984 w_{3}+3577392\right) \\
& \times\left(9 w_{3}^{4}+504 w_{3}^{3}+8504 w_{3}^{2}+78624 w_{3}+1192464\right) \\
& \times\left(9 w_{3}^{4}+576 w_{3}^{3}+19192 w_{3}^{2}+628992 w_{3}+10732176\right)^{2} \\
& \times\left(9 w_{3}^{4}+1152 w_{3}^{3}+58040 w_{3}^{2}+1257984 w_{3}+10732176\right) \\
& \times\left(9 w_{3}^{4}+2736 w_{3}^{3}+164872 w_{3}^{2}+2987712 w_{3}+10732176\right),
\end{aligned}
$$

and six independent points of infinite order with $x$-coordinates:

$$
\begin{aligned}
x_{31}= & 9\left(3 w_{3}^{4}+144 w_{3}^{3}+3160 w_{3}^{2}+157248 w_{3}+3577392\right) \\
& \times\left(3 w_{3}^{4}+1152 w_{3}^{3}+71144 w_{3}^{2}+1257984 w_{3}+3577392\right) \\
& \times\left(9 w_{3}^{4}+576 w_{3}^{3}+19192 w_{3}^{2}+628992 w_{3}+10732176\right)^{2}, \\
x_{32}= & 9\left(3 w_{3}^{4}+144 w_{3}^{3}+3160 w_{3}^{2}+157248 w_{3}+3577392\right) \\
& \times\left(3 w_{3}^{4}+1152 w_{3}^{3}+71144 w_{3}^{2}+1257984 w_{3}+3577392\right) \\
& \times\left(9 w_{3}^{4}+576 w_{3}^{3}+19192 w_{3}^{2}+628992 w_{3}+10732176\right) \\
& \times\left(9 w_{3}^{4}+2736 w_{3}^{3}+164872 w_{3}^{2}+2987712 w_{3}+10732176\right), \\
x_{33}= & 1 / 49\left(3 w_{3}^{4}+144 w_{3}^{3}+3160 w_{3}^{2}+157248 w_{3}+3577392\right) \\
& \times\left(3 w_{3}^{4}+1152 w_{3}^{3}+71144 w_{3}^{2}+1257984 w_{3}+3577392\right) \\
& \times\left(171 w_{3}^{4}+16704 w_{3}^{3}+753128 w_{3}^{2}+18240768 w_{3}+203911344\right)^{2}, \\
x_{34}= & 27\left(w_{3}^{4}+72 w_{3}^{3}+8504 w_{3}^{2}+550368 w_{3}+10732176\right) \\
& \times\left(3 w_{3}^{4}+144 w_{3}^{3}+3160 w_{3}^{2}+157248 w_{3}+3577392\right) \\
& \times\left(9 w_{3}^{4}+504 w_{3}^{3}+8504 w_{3}^{2}+78624 w_{3}+1192464\right) \\
& \times\left(9 w_{3}^{4}+2736 w_{3}^{3}+164872 w_{3}^{2}+2987712 w_{3}+10732176\right), \\
x_{35}= & 27\left(w_{3}^{2}-1092\right)^{2}\left(3 w_{3}^{4}+1152 w_{3}^{3}+71144 w_{3}^{2}+1257984 w_{3}+3577392\right) \\
& \times\left(9 w_{3}^{4}+1152 w_{3}^{3}+58040 w_{3}^{2}+1257984 w_{3}+10732176\right) \\
& \times\left(9 w_{3}^{4}+2736 w_{3}^{3}+164872 w_{3}^{2}+2987712 w_{3}+10732176\right), \\
&
\end{aligned}
$$




$$
\begin{aligned}
x_{36}= & 81\left(w_{3}^{2}+54 w_{3}+1092\right)^{2}\left(3 w_{3}^{4}+144 w_{3}^{3}+3160 w_{3}^{2}+157248 w_{3}+3577392\right) \\
& \times\left(3 w_{3}^{4}+1152 w_{3}^{3}+71144 w_{3}^{2}+1257984 w_{3}+3577392\right) \\
& \times\left(9 w_{3}^{4}+1152 w_{3}^{3}+58040 w_{3}^{2}+1257984 w_{3}+10732176\right) .
\end{aligned}
$$

We also give $y$-coordinates of the points corresponding to $x_{21}$ on (3.5) and $x_{31}$ on (3.6):

$$
\begin{aligned}
y_{21}= & 5760\left(7 w_{2}^{4}+352 w_{2}^{3}-61664 w_{2}^{2}+321024 w_{2}+5822208\right) \\
& \times\left(7 w_{2}^{4}-176 w_{2}^{3}+11680 w_{2}^{2}-160512 w_{2}+5822208\right) \\
& \times\left(w_{2}^{2}+88 w_{2}-1520\right)^{2}\left(5 w_{2}^{2}-264 w_{2}-2736\right)^{2} \\
& \times\left(w_{2}^{4}-44 w_{2}^{3}+4288 w_{2}^{2}-40128 w_{2}+831744\right)^{2}, \\
y_{31}= & 92160 w_{3}^{2}\left(3 w_{3}^{4}+1152 w_{3}^{3}+71144 w_{3}^{2}+1257984 w_{3}+3577392\right) \\
& \times\left(9 w_{3}^{4}+576 w_{3}^{3}+19192 w_{3}^{2}+628992 w_{3}+10732176\right)^{2} \\
& \times\left(3 w_{3}^{4}+144 w_{3}^{3}+3160 w_{3}^{2}+157248 w_{3}+3577392\right)\left(9 w_{3}+364\right)^{2}\left(w_{3}+27\right)^{2} .
\end{aligned}
$$

By factorizing the expressions $a_{62}^{2} b_{63}-a_{63}^{2} b_{62}$ and $a_{62}^{3} y_{31}^{2}-a_{63}^{3} y_{21}^{2}$ we see that for pairs $\left(w_{2}, w_{3}\right)$ satisfying the conditions (3.2) or (3.3) it holds $b_{63} / a_{63}^{2}=b_{62} / a_{62}^{2}$ and $\left(a_{63} / a_{62}\right)^{3}=\left(y_{31} / y_{21}\right)^{2}$. Hence, for such pairs $\left(w_{2}, w_{3}\right)$ the curves (3.5) and (3.6) are isomorphic, where the isomorphism is given by $\phi(x, y)=\left(\frac{a_{63}}{a_{62}} x, \frac{y_{31}}{y_{21}} y\right)$.

In the same way we check that for such pairs $\left(w_{2}, w_{3}\right)$ it holds $x_{31} / a_{63}=$ $x_{21} / a_{62}, x_{32} / a_{63}=x_{22} / a_{62}, x_{33} / a_{63}=x_{23} / a_{62}, x_{34} / a_{63}=x_{24} / a_{62}, x_{35} / a_{63}=$ $x_{25} / a_{62}$, while $x_{36} / a_{63} \neq x_{26} / a_{62}$. Therefore, we have seven points on (3.6) with $x$-coordinates

$$
x_{31}, x_{32}, x_{33}, x_{34}, x_{35}, x_{36}, x_{26} a_{63} / a_{62},
$$

where the last point comes from $\phi\left(x_{26}, y_{26}\right)$. By taking the specialization $\left(w_{2}, w_{3}\right)=\left(-\frac{76}{3}, 26\right)$ we obtain the curve

$$
y^{2}=x^{3}-163531808801344950045916528640000 x^{2}
$$

+ $6680706316011654681276493655189069731350803361465165152256000000 x$

and we checked that seven corresponding points with $x$-coordinates

38540677847903454008558223360000, 178922409809838644555667210240000, 72051389475320867247399895040000,66579605091474988619076835737600 ,

13362426543070313045072805888000,126710845595682509808491456102400

$$
\text { and } 2179385680764224839490312601600
$$

are independent points of infinite order on this curve. By Silverman's specialization theorem ([27, Theorem III.11.4]), we conclude that seven points (3.7) are independent points on (3.6) for infinitely many rational values of $w_{3}$ satisfying the quartic equation (3.4) and the corresponding values $w_{2}$ satisfying 
(3.2) or (3.3). Thus we proved that there are indeed infinitely many elliptic curves induced by rational Diophantine triples with rank $\geq 7$.

Analogous result can be obtained by considering the second and fifth substitution for the parameter $a$. Here the conditions are

$$
\begin{gathered}
9 w_{2}^{2} w_{5}-4 w_{2} w_{5}^{2}-198 w_{2}^{2}+528 w_{5}^{2}+1368 w_{2}-8208 w_{5}=0, \\
11 w_{2}^{2} w_{5}^{2}-171 w_{2}^{2} w_{5}-76 w_{2} w_{5}^{2}+25992 w_{2}+155952 w_{5}-3430944=0,
\end{gathered}
$$

and they lead to the quartic

$$
w_{5}^{4}-1188 w_{5}^{3}+43920 w_{5}^{2}-406296 w_{5}+116964=\square,
$$

which is equivalent to the elliptic curve

$$
y^{2}=x^{3}-x^{2}-124056 x-10126800
$$

with rank equal to 1 , so we again have infinitely many rational solutions. These solutions give seven points on the curve (3.5). By taking the specialization $\left(w_{2}, w_{5}\right)=\left(\frac{6392}{99}, \frac{6392}{99}\right)$ we can check that these seven points are indeed independent, and by Silverman's specialization theorem we conclude that we obtained another infinite family of curves with rank $\geq 7$.

ACKNOWLEDGEMENTS.

The authors would like to thank Luka Lasić and the referee for useful comments on the previous version of this paper. A.D. was supported by the Croatian Science Foundation under the project no. IP-2018-01-1313. He also acknowledges support from the QuantiXLie Center of Excellence, a project co-financed by the Croatian Government and European Union through the European Regional Development Fund - the Competitiveness and Cohesion Operational Programme (Grant KK.01.1.1.01.0004). J.C.P. was supported by the grant: MTM2014-52347-C2-1-R.

\section{REFERENCES}

[1] J. Aguirre, A. Dujella and J. C. Peral, On the rank of elliptic curves coming from rational Diophantine triples, Rocky Mountain J. Math. 42 (2012), 1759-1776.

[2] A. Baker and H. Davenport, The equations $3 x^{2}-2=y^{2}$ and $8 x^{2}-7=z^{2}$, Quart. J. Math. Oxford Ser. (2) 20 (1969), 129-137.

[3] G. Campbell and E. H. Goins, Heron triangles, Diophantine problems and elliptic curves, preprint. http://www.Swarthmore.edu/NatSci/gcampbe1/papers/ heron-Campbell-Goins.pdf

[4] J. Cremona, Algorithms for modular elliptic curves, Cambridge University Press, Cambridge, 1997.

[5] A. Dujella, Diophantine triples and construction of high-rank elliptic curves over $\mathbb{Q}$ with three non-trivial 2-torsion points, Rocky Mountain J. Math. 30 (2000), 157-164.

[6] A. Dujella, There are only finitely many Diophantine quintuples, J. Reine Angew. Math. 566 (2004), 183-214.

[7] A. Dujella, On Mordell-Weil groups of elliptic curves induced by Diophantine triples, Glas. Mat. Ser. III 42(62) (2007), 3-18. 
[8] A. Dujella, What is ... a Diophantine m-tuple?, Notices Amer. Math. Soc. 63 (2016), $772-774$.

[9] A. Dujella, High rank elliptic curves with prescribed torsion, https://web.math.pmf. unizg.hr/ duje/tors/tors.html.

[10] A. Dujella and M. Kazalicki, More on Diophantine sextuples, in: Number Theory Diophantine problems, uniform distribution and applications, Festschrift in honour of Robert F. Tichy's 60th birthday (C. Elsholtz, P. Grabner, Eds.), Springer-Verlag, Berlin, 2017, 227-235.

[11] A. Dujella, M. Kazalicki, M. Mikić and M. Szikszai, There are infinitely many rational Diophantine sextuples, Int. Math. Res. Not. IMRN 2017 (2) (2017), 490-508.

[12] A. Dujella, M. Kazalicki and V. Petričević, There are infinitely many rational Diophantine sextuples with square denominators, J. Number Theory 205 (2019), 340346.

[13] A. Dujella, M. Kazalicki and V. Petričević, Rational Diophantine sextuples containing two regular quadruples and one regular quintuple, Acta Mathematica Spalatensia 1 (2020), 19-27.

[14] A. Dujella and M. Mikić, Rank zero elliptic curves induced by rational Diophantine triples, Rad Hrvat. Akad. Znan. Umjet. Mat. Znan. 24 (2020), 29-37.

[15] A. Dujella and J. C. Peral, High rank elliptic curves with torsion $\mathbb{Z} / 2 \mathbb{Z} \times \mathbb{Z} / 4 \mathbb{Z}$ induced by Diophantine triples, LMS J. Comput. Math. 17 (2014), 282-288.

[16] A. Dujella and J. C. Peral, Elliptic curves induced by Diophantine triples, Rev. R. Acad. Cienc. Exactas Fis. Nat. Ser. A Math. RACSAM 113 (2019), 791-806.

[17] A. Dujella and J. C. Peral, Construction of high rank elliptic curves, J. Geom. Anal., published online: 04 March 2020.

[18] N. D. Elkies, Three lectures on elliptic surfaces and curves of high rank, Lecture notes, Oberwolfach, 2007, arXiv:0709.2908.

[19] N. D. Elkies, Personal communication, 2009.

[20] P. Gibbs, Some rational Diophantine sextuples, Glas. Mat. Ser. III 41(61) (2006), 195-203.

[21] B. He, A. Togbé and V. Ziegler, There is no Diophantine quintuple, Trans. Amer. Math. Soc. 371 (2019), 6665-6709.

[22] E. Herrmann, A. Pethő and H. G. Zimmer, On Fermat's quadruple equations, Abh. Math. Sem. Univ. Hamburg 69 (1999), 283-291.

[23] L. Lasić, Personal communication, 2017.

[24] J.-F. Mestre, Construction de courbes elliptiques sur $\mathbb{Q}$ de rank $\geq 12$, C. R. Acad. Sci. Paris Ser. I 295 (1982), 643-644.

[25] K. Nagao, An example of elliptic curve over $\mathbb{Q}$ with rank $\geq 20$, Proc. Japan Acad. Ser. A Math. Sci. 69 (1993), 291-293.

[26] PARI/GP, version 2.11.1, Bordeaux, 2019, http://pari.math.u-bordeaux.fr.

[27] J. H. Silverman, Advanced Topics in the Arithmetic of Elliptic Curves, SpringerVerlag, New York, 1994

[28] M. Stoll, Diagonal genus 5 curves, elliptic curves over $\mathbb{Q}(t)$, and rational diophantine quintuples, Acta Arith. 190 (2019), 239-261.

[29] R. van Luijk, On Perfect Cuboids, Thesis, Universiteit Utrecht, 2000.

\author{
A. Dujella \\ Department of Mathematics, Faculty of Science \\ University of Zagreb \\ Bijenička cesta 30, 10000 Zagreb \\ Croatia \\ E-mail: duje@math.hr
}


J. C. Peral

Departamento de Matemáticas

Universidad del País Vasco

Aptdo. 644, 48080 Bilbao

Spain

E-mail: juancarlos.peral@ehu.es

Received: 31.5.2020.

Revised: 24.7.2020. 